\title{
Exact Convex Relaxation of OPF for Radial Networks using Branch Flow Model
}

\author{
$\mathrm{Na} \mathrm{Li,} \mathrm{Lijun} \mathrm{Chen,} \mathrm{and} \mathrm{Steven} \mathrm{H.} \mathrm{Low}$
}

\begin{abstract}
The optimal power flow (OPF) problem is generally nonconvex. Recently a second-order cone relaxation for OPF has been proposed using the branch flow model. In this paper, we provide sufficient conditions under which the relaxation is exact, and demonstrate that these conditions hold for a wide class of practical power distribution systems.
\end{abstract}

\section{INTRODUCTION}

In previous work [1], [2], we advocate the use of branch flow models for the design and operation of power systems, including optimal power flow, demand response, and Volt/VAR control. In contrast to bus injection models which focus on nodal variables such as bus current and power injections, branch flow models focus on currents and power flows on individual branches [3], [4]. They have been used mainly for modeling distribution circuits which tend to be radial, but has received far less attention. The optimal power flow (OPF) problem seeks to minimize a certain cost function, such as power loss and generation cost, subject to physical constraints including Kirchoff's laws, thermal constraints, as well as voltage regulation constraints. There has been a great deal of research on OPF since Carpentier's first formulation in 1962 [5]. OPF is generally nonconvex and NP hard, and a large number of optimization algorithms and relaxations have been proposed; see, e.g., [6], [7], [8], [9], [10]. Recently, a semidefinite relaxation (SDR) of OPF is proposed in [11] and a sufficient condition is derived in [12] under which the SDR is exact. This condition is shown to essentially hold in various IEEE test systems. While this line of research has generated a lot of interest, limitations of the SDR have also been studied in [13] using 3, 5, and 7-bus system. Moreover, if SDR fails to provide exact relaxations, the solutions produced by the SDR are physically meaningless in those cases. Remarkably, it turns out that if the network is radial, then the sufficient condition of [12] always holds, provided that the bounds on the power flows satisfy a simple pattern [14], [15], [16]. This is important as almost all distribution systems are radial networks.

Indeed, for radial networks, different convex relaxations have also been studied using branch flow models. The model considered in this paper is first proposed in [3], [4] for the optimal placement and sizing of switched capacitors in distribution circuits for Volt/VAR control. Recasting the model as a set of linear constraints together with a set of quadratic equality constraints, references [17] [1] propose a second-order-cone (SOC) convex relaxation, and prove that the relaxation is

N. Li and S. H. Low are with the Division of Engineering and Applied Science, California Institute of Technology, Pasadena, CA 91125, USA (Emails: \{nali, slow $\} @$ caltech.edu).

L. Chen is with the College of Engineering and Applied Sciences, University of Colorado, Boulder, CO 80309, USA (Email: lijun.chen@ colorado.edu). exact for radial networks, when there are no upper bounds on the loads. See also [18] for an SOC relaxation of a linear approximation of the branch flow model in [3], [4], and [19], [20], [21] for other branch flow models.

Ignoring upper bounds on the load may be unrealistic, e.g., in the context of demand response. In a previous paper [2], we prove that the SOC relaxation is exact for radial networks, provided there are no upper bounds on the voltage magnitudes and some other sufficient conditions hold. Those sufficient conditions however place strong requirements on the impedance of the distribution lines and on the load and generation patterns in the radial network. In this paper, we propose less restrictive sufficient conditions under which the SOC relaxation is exact. As examples, we show that these conditions hold in two distribution circuits of the Southern California Edison (SCE), with high penetration of photovoltaic (PV) generation. Roughly speaking, these sufficient conditions hold in many real distribution systems where $v \sim 1$ p.u., $p, q<1$ p.u. , $r, x<<1$ p.u., and $\frac{r}{x}$ is bounded. Here $v, p, q$ are the bus voltage, real power consumption, and reactive power consumption; and $r, x$ are the resistance and reactance of the distribution lines. Moreover, we provide upper bounds on the voltage magnitudes for the SOC relaxation solutions. This would facilitate the voltage regulation in distribution systems.

The paper is organized as follows. We first present the branch flow model in section II. We then provide in section III sufficient conditions under which the SOC relaxation is exact for radial networks when there are no upper bounds on bus voltage magnitudes. Finally, in section IV, we illustrate these sufficient conditions using two real-world distribution circuits.

\section{PRoblem Formulation}

Due to space limit, we introduce here an abridged version of the branch flow model; see, e.g., [1], [2] for more details.

\section{A. Branch flow model for radial networks}

TABLE I: Notations.

\begin{tabular}{|l|l|}
\hline$V_{i}, v_{i}$ & complex voltage on bus $i$ with $v_{i}=\left|V_{i}\right|^{2}$ \\
\hline$s_{i}=p_{i}+\mathbf{i} q_{i}$ & complex net load on bus $i$ \\
\hline$I_{i j}, \ell_{i j}$ & complex current from buses $i$ to $j$ with $\ell_{i j}=$ \\
& $\left|I_{i j}\right|^{2}$ \\
\hline$S_{i j}=P_{i j}+\mathbf{i} Q_{i j}$ & complex power flowing out from buses $i$ to bus $j$ \\
\hline$z_{i j}=r_{i j}+\mathbf{i} x_{i j}$ & impedance on line $(i, j)$ \\
\hline
\end{tabular}

Consider a radial distribution circuit that consists of a set $N$ of buses and a set $E$ of distribution lines connecting these buses. 
We index the buses in $N$ by $i=0,1, \ldots, n$, and denote a line in $E$ by the pair $(i, j)$ of buses it connects. Bus 0 represents the substation and other buses in $N$ represent branch buses. For each line $(i, j) \in E$, let $I_{i j}$ be the complex current flowing from buses $i$ to $j, z_{i j}=r_{i j}+\mathbf{i} x_{i j}$ the impedance on line $(i, j)$, and $S_{i j}=P_{i j}+\mathbf{i} Q_{i j}$ the complex power flowing from buses $i$ to bus $j$. On each bus $i \in N$, let $V_{i}$ be the complex voltage and $s_{i}$ be the complex net load, i.e., the consumption minus generation. As customary, we assume that the complex voltage $V_{0}$ on the substation bus is given.

The branch flow model was first proposed in [3], [4] to model power flows in a steady state in a radial distribution circuit:

$$
\begin{aligned}
p_{j} & =P_{i j}-r_{i j} \ell_{i j}-\sum_{k:(j, k) \in E} P_{j k}, j=1, \ldots, n \\
q_{j}= & Q_{i j}-x_{i j} \ell_{i j}-\sum_{k:(j, k) \in E} Q_{j k}, j=1, \ldots, n \\
v_{j}= & v_{i}-2\left(r_{i j} P_{i j}+x_{i j} Q_{i j}\right)+\left(r_{i j}^{2}+x_{i j}^{2}\right) \ell_{i j}, \\
\ell_{i j}= & \frac{P_{i j}^{2}+Q_{i j}^{2}}{v_{i}}, \quad(i, j) \in E
\end{aligned}
$$

where $\ell_{i j}:=\left|I_{i j}\right|^{2}, v_{i}:=\left|V_{i}\right|^{2}$, and $p_{i}$ and $q_{i}$ are the real and reactive net loads at node $i$. Equations (1)-(4) define a system of equations in the variables $(P, Q, \ell, v):=$ $\left(P_{i j}, Q_{i j}, \ell_{i j},(i, j) \in E, v_{i}, i=1, \ldots, n\right)$, which do not include phase angles of voltages and currents. Given an $(P, Q, \ell, v)$, these phase angles can be uniquely determined for radial networks. This is not the case for mesh networks; see [1] for exact conditions under which phase angles can be recovered for (an extension of the model here for) mesh networks.

\section{B. Optimal power flow}

Consider the problem of minimizing a cost function over the network where the optimization variables are $p:=$ $\left(p_{1}, \ldots, p_{n}\right), q:=\left(q_{1}, \ldots, q_{n}\right)$, as well as $(P, Q, \ell, v)$. Let

$$
p_{i}:=p_{i}^{c}-p_{i}^{g}, \quad q_{i}:=q_{i}^{c}-q_{i}^{g},
$$

where $p_{i}^{c}$ and $q_{i}^{c}$ are the real and reactive power consumption at node $i$, and $p_{i}^{g}$ and $q_{i}^{g}$ are the real and reactive power generation at node $i$. In addition to power flow equations (1)-(4), we impose the following constraints on power consumption and generation:

$$
\begin{array}{cl}
\underline{p}_{i}^{c} \leq p_{i}^{c} \leq \bar{p}_{i}^{c}, \quad \underline{q}_{i}^{c} \leq q_{i}^{c} \leq \bar{q}_{i}^{c}, \quad i=1, \ldots, n . \\
\underline{p}_{i}^{g} \leq p_{i}^{g} \leq \bar{p}_{i}^{g}, \quad \underline{q}_{i}^{g} \leq q_{i}^{g} \leq \bar{q}_{i}^{g}, & i=1, \ldots, n . \\
f_{i}^{c}\left(p_{i}^{c}, q_{i}^{c}\right) \leq 0, \quad f_{i}^{g}\left(p_{i}^{g}, q_{i}^{g}\right) \leq 0, & i=1, \ldots, n .
\end{array}
$$

Here, equation (7) models additional constraints on $\left(p_{i}^{c}, q_{i}^{c}\right)$ and $\left(p_{i}^{g}, q_{i}^{g}\right)$. For example, for PV generators, $\left(p_{i}^{g}\right)^{2}+\left(q_{i}^{g}\right)^{2} \leq$ $C^{2}$ where $C$ is the capacity of the PV generation [22]. We assume $f_{i}^{c}, f_{i}^{g}$ are convex for all $i=1, \cdots, n$.

Finally, the voltage magnitudes must be maintained to be above certain thresholds:

$$
\underline{v}_{i} \leq v_{i}, \quad i=1, \ldots, n \text {. }
$$

Here we do not impose upper bounds on the voltage magnitudes. However, we derive below upper bounds on the optimal voltage magnitudes.

The objective of the optimal power flow problem is to minimize the power generation costs $C_{i}\left(p_{i}^{g}\right)$, the power losses $r_{i, j} \ell_{i, j}$, and maximize the user utilities $f_{i}\left(p_{i}^{c}\right):{ }^{1}$

OPF:

$$
\begin{aligned}
\min _{P, Q, \ell, v, p, q} & \sum_{i=1}^{n} C_{i}\left(p_{i}^{g}\right)-\sum_{i=1}^{n} f_{i}\left(p_{i}^{c}\right)+\sum_{(i, j) \in E} r_{i, j} \ell_{i, j} \\
\text { s.t. } & (1)-(4),(5)-(8) .
\end{aligned}
$$

OPF is NP hard in general, due to the quadratic equality constraint (4).

\section{EXACT RELAXATION}

\section{A. Second-order cone relaxation}

Following [17], [1], [2], we relax the quadratic equalities in (4) into inequalities and consider the following convex relaxation of OPF.

\section{ROPF:}

$$
\begin{array}{cl}
\min _{P, Q, l, v, p, q} & \sum_{i=1}^{n} C_{i}\left(p_{i}^{g}\right)-\sum_{i=0}^{n} f_{i}\left(p_{i}^{c}\right)+\sum_{(i, j) \in E} r_{i, j} \ell_{i, j} \\
\text { s.t. } & (1)-(3),(5)-(8) \\
& \ell_{i j} \geq \frac{P_{i j}^{2}+Q_{i j}^{2}}{v_{i}}, \quad(i, j) \in E .
\end{array}
$$

Obviously, ROPF provides a lower bound on OPF. It was shown in [17], [1] that this relaxation is exact when there are no upper bounds on the real and reactive power consumptions in (5) but with upper bounds on the voltage magnitudes in (8).

The main result of this paper is a variety of sufficient conditions for exact relaxation when there are no upper bounds on the voltage magnitudes. Given a solution of the relaxed problem ROPF, one can always check if equality is attained in (4). If it is, then the relaxed solution is optimal for the original problem OPF as well. Otherwise, it is not feasible for OPF. Our goal is to develop sufficient conditions for exact relaxation that can be checked without having to solve ROPF first.

\section{B. Sufficient condition for exact relaxation}

We start by developing our results on a simple network, an one-line distribution circuit (main feeder). Then we will extend the results to general radial networks. Due to space limit, all proofs are omitted and can be found in [23].

1) Line networks: For an one-line network, we can abbreviate $r_{i j}, x_{i j}, P_{i j}, Q_{i j}$, and $l_{i j}$ by $r_{i}, x_{i}, P_{i}, Q_{i}$ and $l_{i}$ respectively, as shown in Figure 1. Rewrite the OPF problem in terms of the simplified notations as:

\footnotetext{
${ }^{1} \mathrm{We}$ can also include in the objective function the cost $C_{0}\left(\sum_{(0, j) \in E} P_{0, j}\right)$ on the total power fed into the radial network. This additional term does not change the results of the paper.
} 


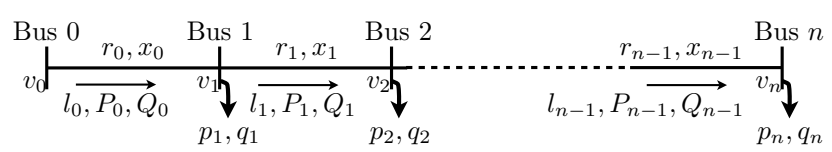

Fig. 1: An one-line distribution network.

\section{LOPF:}

$$
\begin{aligned}
\min _{P, Q, \ell, v, p, q} & \sum_{i=1}^{n} C_{i}\left(p_{i}^{g}\right)-\sum_{i=1}^{n} f_{i}\left(p_{i}^{c}\right)+\sum_{i=0}^{n-1} r_{i} \ell_{i} \\
\text { s.t. } & \frac{P_{i}^{2}+Q_{i}^{2}}{v_{i}}=\ell_{i}, \quad i=0, \cdots, n-1 \\
& P_{i}=P_{i+1}+r_{i} \ell_{i}+p_{i+1}^{c}-p_{i+1}^{g}, \\
& i=0, \cdots, n-1 \\
& Q_{i}=Q_{i+1}+x_{i} \ell_{i}+q_{i+1}^{c}-q_{i+1}^{g} \\
& i=0, \cdots, n-1 \\
& \\
& \begin{array}{r}
i \\
i
\end{array}-v_{i+1}=2\left(r_{i} P_{i}+x_{i} Q_{i}\right)-\left(r_{i}^{2}+x_{i}^{2}\right) \ell_{i}, \\
& i=0, \cdots, n-1
\end{aligned}
$$

The above optimization problem can be relaxed to the following second-order cone program:

\section{RLOPF}

$$
\begin{aligned}
\min _{P, Q, l, v, p, q} & \sum_{i=1}^{n} C_{i}\left(p_{i}^{g}\right)-\sum_{i=1}^{n} f_{i}\left(p_{i}^{c}\right)+\sum_{i=0}^{n-1} r_{i} \ell_{i} \\
\text { s.t. } & (5)-(8),(11)-(13) \\
& \frac{P_{i}^{2}+Q_{i}^{2}}{v_{i}} \leq \ell_{i}, \quad i=0, \cdots, n-1 .
\end{aligned}
$$

The next lemma provides a sufficient condition guaranteeing that RLOPF is an exact relaxation of OPF. For each bus $k \in$ $N \backslash\{0\}$, define $R_{k}:=\sum_{i=0}^{k-1} r_{i}$ and $X_{k}:=\sum_{i=0}^{k-1} x_{i}$ as the cumulative resistance and reactance from the feeder to bus $k$. Also define $[a]^{+}=\max (a, 0)$.

Lemma 1. Any optimal solution $(P, Q, \ell, v, p, q)$ of $R L O P F$ is also optimal for $L O P F$, provided that for each $k \in N \backslash\{0\}$ the following condition holds: for all $i<k$,

$$
\begin{aligned}
v_{i}>2 \max & \left(-X_{k}\left[\frac{r_{k}}{x_{k}}-\frac{R_{k}}{X_{k}}\right]^{+} P_{i},\right. \\
& \left.-R_{k}\left[\frac{x_{k}}{r_{k}}-\frac{X_{k}}{R_{k}}\right]^{+} Q_{i}\right) .
\end{aligned}
$$

Moreover, for each node $i \in N \backslash\{0\}$, the voltage is upperbounded by: ${ }^{2}$

$$
v_{i} \leq v_{0}-2 \sum_{k=0}^{i-1}\left(r_{k}\left(P_{k}-r_{k} \ell_{k}\right)+x_{k}\left(Q_{k}-x_{k} \ell_{k}\right)\right) .
$$

The condition (15) in Lemma 1 is not checkable before solving RLOPF as it involves a solution $(P, Q, \ell, v, p, q)$ of

\footnotetext{
${ }^{2}$ Note that $P_{k}-r_{k} \ell_{k}$ and $Q_{k}-x_{k} \ell_{k}$ are the real and reactive power received by bus $k+1$ from bus $k$.
}

RLOPF. We now provide a checkable condition by bounding $v_{i}, P_{i}, Q_{i}$ in terms of system parameters $\underline{p}_{i}, \bar{p}_{i}, \underline{q}_{i}, \bar{q}_{i}, \underline{v}_{i}$. Define

$$
\underline{P}_{i}^{n o m} \triangleq \sum_{j=i+1}^{n}\left(\underline{p}_{j}^{c}-\bar{p}_{j}^{g}\right) ; \underline{Q}_{i}^{n o m} \triangleq \sum_{j=i+1}^{n}\left(\underline{q}_{j}^{c}-\bar{q}_{j}^{g}\right) .
$$

Using (11)-(12) we can iteratively derive that for any $i \in N$,

$$
P_{i} \geq P_{i}-r_{i} \ell_{i} \geq \underline{P}_{i}^{n o m},
$$

and

$$
Q_{i} \geq Q_{i}-r_{i} \ell_{i} \geq \underline{Q}_{i}^{n o m} .
$$

Combining the above two inequalities with Lemma 1 give the following result.

Theorem 2. Any optimal solution of RLOPF is also optimal for $L O P F$, provided that for each $k \in N \backslash\{0\}$ the following condition holds: for all $i<k$,

$$
\begin{aligned}
\underline{v}_{i}>2 \max ( & X_{k}\left[\frac{r_{k}}{x_{k}}-\frac{R_{k}}{X_{k}}\right]^{+} \underline{P}_{i}^{n o m}, \\
& \left.-R_{k}\left[\frac{x_{k}}{r_{k}}-\frac{X_{k}}{R_{k}}\right]^{+} \underline{Q}_{i}^{n o m}\right) .
\end{aligned}
$$

Moreover, for each node $i \in N \backslash\{0\}$, the voltage is upperbounded by:

$$
v_{i} \leq v_{0}-2 \sum_{k=0}^{i-1}\left(r_{k} \underline{P}_{k}^{n o m}+x_{k} \underline{Q}_{k}^{n o m}\right) .
$$

Since $X_{k}\left[\frac{r_{k}}{x_{k}}-\frac{R_{k}}{X_{k}}\right]^{+} \geq 0, R_{k}\left[\frac{x_{k}}{r_{k}}-\frac{X_{k}}{R_{k}}\right]^{+} \geq 0$ for each $k=1, \cdots, n$, and $\underline{v}_{i}>0$ for each $i \in N$, we have the following special cases:

(i) If $\underline{P}_{i}^{\text {nom }}>0$ and $\underline{Q}_{i}^{\text {nom }}>0$ for all $i \in N \backslash\{0\}$, then the right-hand side of (16) is always non-positive, which implies that (16) is always satisfied. Hence the relaxation is exact provided that both the real and reactive powers do not flow backward.

(ii) If $\frac{x_{k}}{r_{k}} \leq \frac{X_{k}}{R_{k}}$ for any $k=1, \cdots, n$, then $\left[\frac{x_{k}}{r_{k}}-\frac{X_{k}}{R_{k}}\right]^{+}=0$. Condition (16) reduces to the following condition:

$$
\underline{v}_{i}>2 \max \left(-X_{k}\left[\frac{r_{k}}{x_{k}}-\frac{R_{k}}{X_{k}}\right] \underline{P}_{i}^{n o m}\right), \forall i<k .
$$

This condition is always satisfied if $\underline{P}_{i}^{\text {nom }} \geq 0$ for all $i \in N$. Therefore the relaxation is exact provided real powers do not flow backward.

(iii) If $\frac{x_{k}}{r_{k}} \geq \frac{X_{k}}{R_{k}}$ for all $k=1, \cdots, n$, then the same argument as above shows that the relaxation is exact provided reactive powers do not flow backward, i.e., if $Q_{i}^{\text {nom }} \geq 0$ for all $i \in N$.

(iv) $\frac{{ }^{\prime}}{i} \frac{x_{k}}{r_{k}}=\frac{X_{k}}{R_{x}}$ for all $k=1, \cdots, n$, then $\left[\frac{r_{k}}{x_{k}}-\frac{R_{k}}{X_{k}}\right]^{+}=0$ and $\left[\frac{x_{k}}{r_{k}}-\frac{X_{k}}{R_{k}}\right]^{+}=0$. Therefore, condition (16) reduces to: $v_{i}>0$ for all $i \in N$. This is always satisfied.

These four special cases are the main results in our previous work [2]. See [2] for further discussion on their implications.

The conditions in these special cases are more stringent than (16) and may not hold in practice. The sufficient condition 
(16) depends only on how $\underline{v}_{i}$ compare with the products of resistances (reactances) and real (reactive) powers. In practice, $|V| \sim 1$ p.u., $r, x<<1$ p.u., $\frac{r}{x} \sim[0.1,10]$, and $p, q<1$ p.u.. As we show in Section IV, condition (16) usually holds when the system parameters are in these ranges.

2) General radial networks: We now extend Lemma 1 and Theorem 2 to general radial distribution circuits. Given a radial network:

- For each node $k$, denote the unique path from root 0 to node $k$ by $\mathcal{P}_{k} \triangleq\{(i, j):(i, j) \in$ $E$ is on the path from root 0 to node $k\}$.

- Define the cumulative resistance and reactance from root 0 to node $k$ as $R_{k} \triangleq \sum_{(i, j) \in \mathcal{P}_{k}} r_{i, j}$ and $X_{k} \triangleq$ $\sum_{(i, j) \in \mathcal{P}_{k}} x_{i, j}$.

It is straightforward to extend Lemma 1 to the case of general radial networks.

Lemma 3. Any optimal solution $(P, Q, \ell, v, p, q)$ of $R O P F$ is also optimal for $O P F$, provided that for each $(k, l) \in E$ the following condition holds: for all $(i, j) \in \mathcal{P}_{k}$,

$$
\begin{aligned}
v_{i}>2 \max & -X_{k}\left[\frac{r_{k, l}}{x_{k, l}}-\frac{R_{k}}{X_{k}}\right]^{+} P_{i, j}, \\
& \left.-R_{k}\left[\frac{x_{k, l}}{r_{k, l}}-\frac{X_{k}}{R_{k}}\right]^{+} Q_{i, j}\right) .
\end{aligned}
$$

Moreover, for each node $i \in N \backslash\{0\}$, the voltage is upperbounded by:

$v_{i} \leq v_{0}-2 \sum_{(j, k) \in \mathcal{P}_{i}}\left(r_{j, k}\left(P_{j, k}-r_{j, k} \ell_{j, k}\right)+x_{j, k}\left(Q_{j, k}-x_{i, j} \ell_{j, k}\right)\right)$.

Similarly, this lemma involves a solution $v_{i}, P_{i, j}, Q_{i, j}$ of ROPF. For a sufficient condition that does not require solving ROPF first, define

$$
\underline{P}_{j}^{n o m} \triangleq \sum_{i \in D(j)}^{n}\left(\underline{p}_{i}^{c}-\bar{p}_{i}^{g}\right) ; \quad \underline{Q}_{j}^{n o m} \triangleq \sum_{i \in D(j)}^{n}\left(\underline{q}_{i}^{c}-\bar{q}_{i}^{g}\right),
$$

for each $j=1, \cdots, n$. Here $D(j)$ is the set of all the descendants of $j$ including $j$ itself. ${ }^{3}$ Note that for any $(i, j) \in E$,

$$
\begin{gathered}
P_{i, j} \geq P_{i, j}-r_{i, j} \ell_{i, j} \geq \underline{P}_{j}^{n o m}, \\
Q_{i, j} \geq Q_{i, j}-x_{i, j} \ell_{i, j} \geq \underline{Q}_{j}^{n o m} .
\end{gathered}
$$

Lemma 3 then implies the following extension of Theorem 2 .

Theorem 4. Any optimal solution of ROPF is also optimal for $O P F$, provided that for each $(k, l) \in E$ the following condition holds: for all $(i, j) \in \mathcal{P}_{k}$,

$$
\begin{array}{r}
\underline{v}_{i}>2 \max \left(-X_{k}\left[\frac{r_{k, l}}{x_{k, l}}-\frac{R_{k}}{X_{k}}\right]^{+} \underline{P}_{j}^{n o m},\right. \\
\left.-R_{k}\left[\frac{x_{k, l}}{r_{k, l}}-\frac{X_{k}}{R_{k}}\right]^{+} \underline{Q}_{j}^{n o m}\right) .
\end{array}
$$

\footnotetext{
${ }^{3}$ A rigorous definition of $D(j)$ is: $D(j) \triangleq\{l \in N$ : there exist a sequence of nodes, $j_{0}, j_{1}, \cdots, j_{m}$, such that $j_{0}=j, j_{m}=$ $l$, and $\left(j_{i}, j_{i+1}\right) \in E, \forall i=0, \ldots, m-1$, where $\left.m \geq 0\right\}$.
}

Moreover, for each node $i \in N \backslash\{0\}$, the voltage is upperbounded by:

$$
v_{i} \leq v_{0}-2 \sum_{(j, k) \in \mathcal{P}_{i}}\left(r_{j, k} \underline{P}_{k}^{n o m}+x_{j, k} \underline{Q}_{k}^{n o m}\right) .
$$

The above theorem requires checking condition (17) for each $(k, l) \in E$ and for all $(i, j) \in \mathcal{P}_{k}$. We can derive a simpler though more conservative sufficient condition for

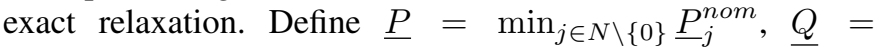
$\min _{j \in N \backslash\{0\}} \underline{Q}_{j}^{n o m}$, and $\underline{v}=\min _{i \in N} \underline{v}_{i}$. Note that for any $(i, j) \in E, P_{i, j} \geq \underline{P}, Q_{i, j} \geq \underline{Q}$, and $v_{i} \geq \underline{v}$.

Corollary 5. Any optimal solution of ROPF is also optimal for $O P F$, provided:

$$
\begin{array}{r}
\underline{v}>-2 \min \\
\left(\underline{P} \cdot \max _{(k, l) \in E}\left(X_{k}\left[\frac{r_{k, l}}{x_{k, l}}-\frac{R_{k}}{X_{k}}\right]^{+}\right),\right. \\
\left.\underline{Q} \cdot \max _{(k, l) \in E}\left(R_{k}\left[\frac{x_{k, l}}{r_{k, l}}-\frac{X_{k}}{R_{k}}\right]^{+}\right)\right) .
\end{array}
$$

Moreover, for each node $i \in N \backslash\{0\}$, the voltage is upperboudned by:

$$
v_{i} \leq v_{0}-2 R_{i} \underline{P}-2 X_{i} \underline{Q} .
$$

Corollary 5 provides a condition which is much easier to check but more restrictive. Nonetheless, since $|V| \sim 1$ p.u., $r, x<<1$ p.u., and $p, q<1$ p.u. in practice, condition (18) holds for both a 47-bus distribution circuit and a 56-bus distribution circuit of the Southern California Edison (SCE), as show in Section IV.

Finally, the following corollary summarizes the special cases we have discussed in Section III-B1.

Corollary 6. Any optimal solution of ROPF is also optimal for $O P F$, provided that one of the following conditions holds:

1) For each $j \in N \backslash\{0\}, \underline{P}_{j}^{\text {nom }} \geq 0, \underline{Q}_{j}^{\text {nom }} \geq 0$;

2) For all $(i, j) \in E, \frac{r_{i, j}}{x_{i, j}} \geq \frac{R_{i}}{X_{i}}, \underline{P}_{j}^{n o m} \geq 0$;

3) For all $(i, j),(j, k) \in E, \frac{r_{j, k}}{x_{j, k}} \geq \frac{r_{i, j}}{x_{i, j}}, \underline{P}_{j}^{\text {nom }} \geq 0$;

4) For all $(i, j) \in E, \frac{x_{i, j}}{r_{i, j}} \geq \frac{X_{i}}{R_{i}}, \underline{Q}_{j}^{n o m} \geq 0$;

5) For all $(i, j),(j, k) \in E, \frac{x_{j, k}}{r_{j, k}} \geq \frac{x_{i, j}}{r_{i, j}}, \underline{Q}_{j}^{\text {nom }} \geq 0$;

6) For all $(i, j),(j, k) \in E, \frac{r_{j, k}}{x_{j, k}}=\frac{r_{i, j}}{x_{i, j}}$.

\section{CASE STUdY}

In this section we evaluate these conditions for exact relaxation for two distribution circuits of SCE with high penetration of photovoltaic (PV) generation [17], [24]. Figure 2 shows a 47-bus distribution circuit and Table II lists the network data including line impedances, peak MVA demand of loads, and the nameplate capacity of the shunt capacitors and the photovoltaic generations [17]. Note that Bus 1 indicates the substation, and there are 5 photovoltaic (PV) generators located on buses 13, 17, 19, 23 and 24. See [24] for the schematic diagram and network data for another circuit with 56 buses. 


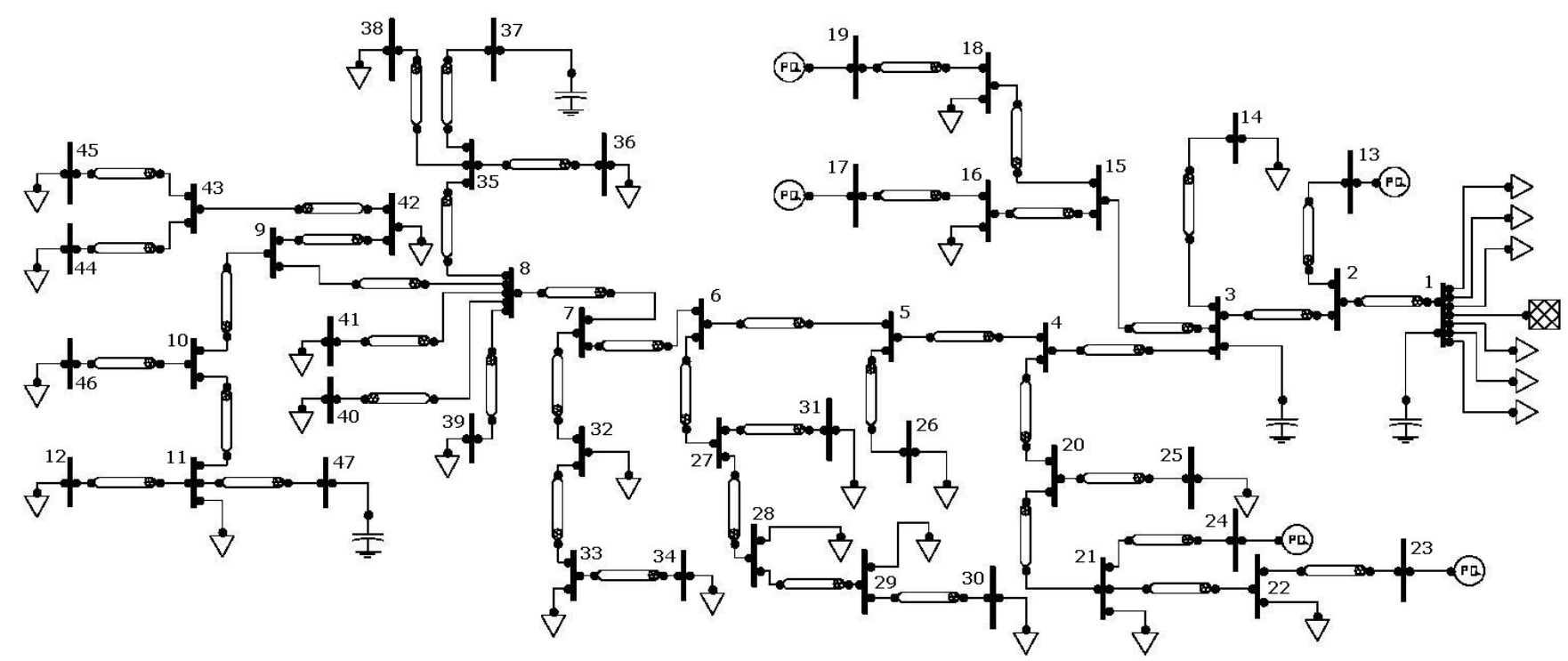

Fig. 2: Schematic diagram of two SCE distribution systems.

TABLE II: Network of Figure 2: Line impedances, peak spot load KVA, Capacitors and PV generation's nameplate ratings.

\begin{tabular}{|c|c|c|c|c|c|c|c|c|c|c|c|c|c|c|c|c|c|}
\hline \multicolumn{18}{|c|}{ Network Data } \\
\hline \multicolumn{4}{|c|}{ Line Data } & \multicolumn{4}{|c|}{ Line Data } & \multicolumn{4}{|c|}{ Line Data } & \multicolumn{2}{|c|}{ Load Data } & \multicolumn{2}{|c|}{ Load Data } & \multicolumn{2}{|c|}{ PV Generators } \\
\hline $\begin{array}{l}\text { From } \\
\text { Bus. }\end{array}$ & $\begin{array}{l}\text { To } \\
\text { Bus. }\end{array}$ & $\begin{array}{l}\mathrm{R} \\
(\Omega)\end{array}$ & $\begin{array}{l}\mathrm{X} \\
(\Omega)\end{array}$ & $\begin{array}{l}\text { From } \\
\text { Bus. }\end{array}$ & $\begin{array}{l}\text { To } \\
\text { Bus. }\end{array}$ & $\begin{array}{l}\mathrm{R} \\
(\Omega)\end{array}$ & $\begin{array}{l}\mathrm{X} \\
(\Omega)\end{array}$ & \begin{tabular}{l|} 
From \\
Bus.
\end{tabular} & $\begin{array}{l}\text { To } \\
\text { Bus. }\end{array}$ & $\begin{array}{l}\mathrm{R} \\
(\Omega)\end{array}$ & $\begin{array}{l}\mathrm{X} \\
(\Omega)\end{array}$ & $\begin{array}{l}\text { Bus } \\
\text { No. }\end{array}$ & $\begin{array}{l}\text { Peak } \\
\text { MVA }\end{array}$ & $\begin{array}{l}\text { Bus } \\
\text { No. }\end{array}$ & $\begin{array}{l}\text { Peak } \\
\text { MVA }\end{array}$ & $\begin{array}{l}\text { Bus } \\
\text { No. }\end{array}$ & $\begin{array}{l}\text { Nameplate } \\
\text { Capacity }\end{array}$ \\
\hline 1 & 2 & 0.259 & 0.808 & 8 & 41 & 0.107 & 0.031 & 21 & 22 & 0.198 & 0.046 & 1 & 30 & 34 & 0.2 & & \\
\hline 2 & 13 & 0 & 0 & 8 & 35 & 0.076 & 0.015 & 22 & 23 & 0 & 0 & 11 & 0.67 & 36 & 0.27 & 13 & $1.5 \mathrm{MW}$ \\
\hline 2 & 3 & 0.031 & 0.092 & 8 & 9 & 0.031 & 0.031 & 27 & 31 & 0.046 & 0.015 & 12 & 0.45 & 38 & 0.45 & 17 & $0.4 \mathrm{MW}$ \\
\hline 3 & 4 & 0.046 & 0.092 & 9 & 10 & 0.015 & 0.015 & 27 & 28 & 0.107 & 0.031 & 14 & 0.89 & 39 & 1.34 & 19 & $1.5 \mathrm{MW}$ \\
\hline 3 & 14 & 0.092 & 0.031 & 9 & 42 & 0.153 & 0.046 & 28 & 29 & 0.107 & 0.031 & 16 & 0.07 & 40 & 0.13 & 23 & $1 \mathrm{MW}$ \\
\hline 3 & 15 & 0.214 & 0.046 & 10 & 11 & 0.107 & 0.076 & 29 & 30 & 0.061 & 0.015 & 18 & 0.67 & 41 & 0.67 & 24 & $2 \mathrm{MW}$ \\
\hline 4 & 5 & 0.107 & 0.183 & 11 & 47 & 0.031 & 0.015 & 33 & 34 & 0.031 & 0 & 22 & 2.23 & 44 & 0.45 & & Capacitors \\
\hline 5 & 26 & 0.061 & 0.015 & 11 & 12 & 0.076 & 0.046 & 35 & 36 & 0.076 & 0.015 & 25 & 0.45 & 45 & 0.2 & Bus & Nameplate \\
\hline 5 & 6 & 0.015 & 0.031 & 15 & 18 & 0.046 & 0.015 & 35 & 37 & 0.076 & 0.046 & 26 & 0.2 & 46 & 0.45 & No. & Capacity \\
\hline 6 & 27 & 0.168 & 0.061 & 15 & 16 & 0.107 & 0.015 & 35 & 38 & 0.107 & 0.015 & 28 & 0.13 & & & & \\
\hline 6 & 7 & 0.031 & 0.046 & 16 & 17 & 0 & 0 & 42 & 43 & 0.061 & 0.015 & 29 & 0.13 & Base & $(\mathrm{KV})=12.35$ & 1 & $6000 \mathrm{KVAR}$ \\
\hline 7 & 32 & 0.076 & 0.015 & 18 & 19 & 0 & 0 & 43 & 44 & 0.061 & 0.015 & 30 & 0.2 & & $\mathrm{~A}=1000$ & 3 & $1200 \mathrm{KVAR}$ \\
\hline 7 & 8 & 0.015 & 0.015 & 20 & 21 & 0.122 & 0.092 & 43 & 45 & 0.061 & 0.015 & 31 & 0.07 & Subs & ltage $=12.35$ & 37 & $1800 \mathrm{KVAR}$ \\
\hline 8 & 40 & 0.046 & 0.015 & 20 & 25 & 0.214 & 0.046 & & & & & 32 & 0.13 & & & 47 & $1800 \mathrm{KVAR}$ \\
\hline 8 & 39 & 0.244 & 0.046 & 21 & 24 & 0 & 0 & & & & & 33 & 0.27 & & & & \\
\hline
\end{tabular}

\section{A. Verifying sufficient conditions}

We verify that the condition in Corollary 5 holds in both circuits. To calculate $\underline{P}$ and $Q$, we only need values for lower bounds of $\left(p_{i}^{c}, q_{i}^{c}\right)$ and upper bounds of $\left(p_{i}^{g}, q_{i}^{g}\right)$ :

- For load buses, we set $\underline{p}_{i}^{c}$ to be 0 and set $\underline{q}_{i}^{c}$ to be the negative of peak MVA value.

- For PV generators, we set $\left(\bar{p}_{i}^{g}, \bar{q}_{i}^{g}\right)$ to be the generators' capacities.

- For shunt capacitors, we treat them as reactive power generators and set $\bar{q}_{i}^{g}$ to be their shunt capacities.

47-bus circuit: We calculate the following values:

- $\underline{P}=-6.4 \mathrm{MW} ; \quad Q=-22.5 \mathrm{MW}$

- $\max _{(k, l) \in E}\left(X_{k}\left[\frac{r_{k, l}}{x_{k, l}}-\frac{R_{k}}{X_{k}}\right]^{+}\right)=8.5649 \Omega$;

- $\max _{(k, l) \in E}\left(R_{k}\left[\frac{x_{k, l}}{r_{k, l}}-\frac{X_{k}}{R_{k}}\right]^{+}\right)=0.9776 \Omega$.

The right-hand side of inequality (18) is 109.6311. Since the nominal voltage is $12.35 \mathrm{KV}$, as long as the voltage magnitudes are maintained above $85 \%$ of the nominal value (which they are in practice), i.e., $\underline{v}_{i} \geq(12.35 \times 0.85)^{2}=110.1975$ for each bus $i$, then (18) holds and ROPF is an exact relaxation of OPF. Moreover, (19) provides an upper bound for the voltage magnitude on each bus: $\max _{i \in N} \sqrt{v_{0}-2 R_{i} \underline{P}-2 X_{i} \underline{Q}}=$ $15.1594 \mathrm{KV}$.

56-bus circuit: Similarly, we calculate the following:

- $\underline{P}=-5 \mathrm{MV} ; \quad Q=-11.435 \mathrm{MV}$

- $\max _{(k, l) \in E}\left(X_{k}\left[\frac{r_{k, l}}{x_{k, l}}-\frac{R_{k}}{X_{k}}\right]^{+}\right)=10.8583 \Omega$;

- $\max _{(k, l) \in E}\left(R_{k}\left[\frac{x_{k, l}}{r_{k, l}}-\frac{X_{k}}{R_{k}}\right]^{+}\right)=5.9590 \Omega$.

The right-hand side of inequality (18) is 108.5383 . Since the nominal voltage is $12 \mathrm{KV}$, as long as the voltage magnitudes are maintained above $87.5 \%$ of the nominal value (which they are in practice), i.e., $\underline{v}_{i} \geq(12 \times 0.875)^{2}=110.25$ for each bus $i$, then (18) holds and ROPF is an exact relaxation of OPF. Moreover, (19) provides an upper bound for the voltage magnitude on each bus: $\max _{i \in N} \sqrt{v_{0}-2 R_{i} \underline{P}-2 X_{i} \underline{Q}}=$ $18.1146 \mathrm{KV}$. 
Remark 1. All the above analysis is worst-case. In reality, $p_{i}^{c}$ and $\underline{q}_{i}^{c}$ tend to be larger than the values we used above, and $\underline{p}_{i}^{g}$ and $\underline{q}_{i}^{g}$ smaller. This implies larger $(\underline{P}, Q)$ and smaller values for the right-hand side of inequality (18) and of inequality (19) than the values we have calculated above. Thus the sufficient condition in Corollary 5 is easier to meet and the voltage upper bound is tighter than implied by the worst-case analysis. This is further verified in the simulations below.

Remark 2. Condition (17) in Theorem 4 can be used as a rule of thumb for designing distribution circuits that will ensure that ROPF is an exact relaxation of OPF. Specifically, if the distribution lines are more uniform, namely that distribution lines have closer resistance to reactance ratios $\frac{r}{x}$, or if the distribution lines have smaller resistance and reactance, then condition (17) is easier to satisfy.

\section{B. Simulation}

We have also solved Problem ROPF using the CVX toolbox [25]. In the simulation:

- for each load bus, we set $\bar{p}_{i}^{c}$ and $\bar{q}_{i}^{c}$ as the peak MVA value, $\underline{p}_{i}^{c}, \underline{q}_{i}^{c}$ as half of the peak MVA value. We use utility functions of the form $-a_{i}\left(p_{i}-\bar{p}_{i}^{c}\right)^{2}+b_{i}$ where $a_{i}$, $b_{i}$ are drawn randomly from $[2.5,5]$;

- for each PV generator, we set $\left(\bar{p}_{i}^{g}, \bar{q}_{i}^{g}\right)$ as the generator's capacity value, $\underline{p}_{i}^{g}, \underline{q}_{i}^{g}$ as 0 . We use cost functions of the form $a_{i} p_{i}^{2}$ where $a_{i}$ are drawn randomly from $[2.5,5]$;

- for each shunt capacitor, we treat them as reactive power generators and set $\bar{p}_{i}^{c}=\underline{p}_{i}^{c}=\underline{q}_{i}^{c}=0$ and $\bar{q}_{i}^{g}$ as their shunt capacities;

- we use a cost $C_{0}\left(P_{0}\right):=C_{0}\left(\sum_{j:(0, j) \in E} P_{0, j}\right)$ on the total power fed into this distribution system of the form $a_{0} P_{0}^{2}+b_{0} P_{0}$ with $a_{0}=0.1, b_{0}=0.1$.

After solving ROPF using CVX toolbox for both the 47-bus system and the 56-bus system, we verify that the solutions of ROPF satisfy the equality constraint (4) and are therefore optimal for OPF. This implies that ROPF indeed is an exact relaxation of OPF for both distribution circuits. Moreover, in each case, the maximum voltage magnitude of the optimal solution is $12.35 \mathrm{KV}$ which is the voltage magnitude of feeder and much less than the upper bound we provided in Section IV-A.

\section{CONCLUSION}

We have studied the second-order cone relaxation of the optimal power flow problem in radial networks using the branch flow model. We provide sufficient conditions under which the relaxation is exact when there are no upper bounds on the voltage magnitudes. We have verified that these conditions are satisfied in two real-world distribution circuits.

\section{ACKNOWLEDGEMENT}

The authors would like to thank Lingwen Gan for insightful discussion on this topic. This work is supported by NSF NetSE grant CNS 0911041, ARPA-E grant DE-AR0000226, Southern California Edison, National Science Council of Taiwan, R.O.C. grant, NSC 101- 3113-P-008-001, the Caltech Resnick Institute, and the Okawa Foundation.

\section{REFERENCES}

[1] M. Farivar and S. Low, "Branch flow model: Relaxations and convexification," arXiv:1204.4865v2, 2012.

[2] L. Gan, N. Li, U. Topcu, and S. Low, "Branch flow model for radial networks: convex relaxation," in Proceedings of the 51st IEEE Conference on Decision and Control, 2012.

[3] M. E. Baran and F. F. Wu, "Optimal Capacitor Placement on radial distribution systems," IEEE Trans. Power Delivery, vol. 4, no. 1, pp. 725-734, 1989.

[4] — - "Optimal Sizing of Capacitors Placed on A Radial Distribution System," IEEE Trans. Power Delivery, vol. 4, no. 1, pp. 735-743, 1989.

[5] J. Carpentier, "Contribution to the economic dispatch problem," Bulletin de la Societe Francoise des Electriciens, vol. 3, no. 8, pp. 431-447, 1962, in French.

[6] J. A. Momoh, Electric Power System Applications of Optimization, ser Power Engineering, H. L. Willis, Ed. Markel Dekker Inc.: New York, USA, 2001.

[7] M. Huneault and F. D. Galiana, "A survey of the optimal power flow literature," IEEE Trans. on Power Systems, vol. 6, no. 2, pp. 762-770, 1991.

[8] J. A. Momoh, M. E. El-Hawary, and R. Adapa, "A review of selected optimal power flow literature to 1993. Part I: Nonlinear and quadratic programming approaches," IEEE Trans. on Power Systems, vol. 14, no. 1 , pp. 96-104, 1999.

[9] _ "A review of selected optimal power flow literature to 1993 Part II: Newton, linear programming and interior point methods," IEEE Trans. on Power Systems, vol. 14, no. 1, pp. $105-111,1999$.

[10] K. S. Pandya and S. K. Joshi, "A survey of optimal power flow methods," J. of Theoretical and Applied Information Technology, vol. 4, no. 5, pp. 450-458, 2008

[11] X. Bai, H. Wei, K. Fujisawa, and Y. Wang, "Semidefinite programming for optimal power flow problems," Int'l J. of Electrical Power \& Energy Systems, vol. 30, no. 6-7, pp. 383-392, 2008.

[12] J. Lavaei and S. Low, "Zero duality gap in optimal power flow problem," IEEE Trans. on Power Systems, vol. 27, no. 1, pp. 92-107, 2012

[13] B. Lesieutre, D. Molzahn, A. Borden, and C. L. DeMarco, "Examining the limits of the application of semidefinite programming to power flow problems," in Proc. Allerton Conference, 2011.

[14] S. Bose, D. Gayme, S. H. Low, and K. M. Chandy, "Optimal power flow over tree networks," in Proc. Allerton Conf. on Comm., Ctrl. and Computing, October 2011.

[15] B. Zhang and D. Tse, "Geometry of feasible injection region of power networks," Arxiv preprint arXiv:1107.1467, 2011.

[16] S. Sojoudi and J. Lavaei, "Network topologies guaranteeing zero duality gap for optimal power flow problem," 2012.

[17] M. Farivar, C. R. Clarke, S. H. Low, and K. M. Chandy, "Inverter var control for distribution systems with renewables," in Proceedings of IEEE SmartGridComm Conference, October 2011.

[18] J. A. Taylor, "Conic optimization of electric power systems," Ph.D. dissertation, MIT, June 2011.

[19] R. Cespedes, "New method for the analysis of distribution networks," IEEE Trans. Power Del., vol. 5, no. 1, pp. 391-396, January 1990.

[20] A. G. Expósito and E. R. Ramos, "Reliable load flow technique for radial distribution networks," IEEE Trans. Power Syst., vol. 14, no. 13, pp. 1063-1069, August 1999.

[21] R. Jabr, "Radial Distribution Load Flow Using Conic Programming," IEEE Trans. on Power Systems, vol. 21, no. 3, pp. 1458-1459, 2006.

[22] K. Turitsyn, P. Sŭlc, S. Backhaus, and M. Chertkov, "Distributed control of reactive power flow in a radial distribution circuit with high photovoltaic penetration," in Proc. of IEEE PES General Meeting, July 2010, pp. $1-6$.

[23] N. Li, L. Chen, and S. Low, "Exact convex relaxation for radial networks using branch flow models," Technical Report, Available at: http://www. cds.caltech.edu/ nali/, Tech. Rep., 2012.

[24] M. Farivar, R. Neal, C. Clarke, and S. Low, "Optimal inverter var control in distribution systems with high pv penetration," in IEEE Power and Energy Society General Meeting, San Diego, CA, July 2012.

[25] M. Grant, S. Boyd, and Y. Ye, "Cvx user guide," Technical Report, Available at: http://cvxr.com/cvx/cvx_usrguide.pdf, Tech. Rep., 2009. 